\title{
Early COVID-19 Vaccine Hesitancy Characteristics in Mothers Following Bariatric Surgery
}

\author{
Heather Strong ${ }^{1}\left[\right.$ ] Jennifer Reiter-Purtill ${ }^{1} \cdot$ Taylor Howarth $^{1} \cdot$ Lisa West-Smith ${ }^{2} \cdot$ Meg H. Zeller ${ }^{1,3}$
}

Received: 13 August 2021 / Revised: 24 December 2021 / Accepted: 29 December 2021 / Published online: 8 January 2022

(c) The Author(s), under exclusive licence to Springer Science+Business Media, LLC, part of Springer Nature 2022

\begin{abstract}
Background Obesity has played a central role in heightened coronavirus disease 2019 (COVID-19) risk and vaccine response. COVID-19 vaccine intention among those with a history of severe obesity, specifically those who have undergone bariatric surgery, has not been described. This study aims to examine early COVID-19 vaccine intention among mothers with a history of severe obesity who underwent bariatric surgery.

Methods Sixty-four mothers $\left(M_{\text {age }}=39.3\right.$ years $)$ who underwent bariatric surgery $\left(M_{\text {time since surgery }}=19.6\right.$ months $)$ completed surveys online (November 2020-February 2021). Information obtained included their COVID-19 vaccine intention (vaccine ready, undecided, vaccine opposed). Analyses examined group differences in demographics, body mass index $(\mathrm{BMI}=\mathrm{kg} /$ $\mathrm{m}^{2}$ ), knowledge of obesity-related COVID-19 risk, flu vaccination history, general beliefs about vaccine safety/effectiveness, and factors increasing confidence/motivation to obtain a COVID-19 vaccine.

Results Thirty-six $(56.3 \%)$ mothers had severe obesity ( $\geq$ Class II $\left.\left[\mathrm{BMI}=\geq 35 \mathrm{~kg} / \mathrm{m}^{2}\right]\right)$. The majority were vaccine hesitant (undecided $[n=28 ; 43.8 \%]$; vaccine opposed $[n=15 ; 23.4 \%]$ ). Compared to the vaccine-ready group, vaccine-hesitant groups were younger $(p<.05)$. For the vaccine opposed, recent flu vaccination rates $(p=.012)$ and general belief that vaccines are safe $(p=.028)$ were lower than expected. Among hesitant participants, no reported side effects and the health of self and others were endorsed as top factors increasing vaccine confidence and motivation respectively.

Conclusions While preliminary, the prominence of early vaccine hesitancy in this sample of mothers who have undergone bariatric surgery, with most persisting with severe obesity, indicates a subgroup at high risk. Factors to address through targeted messaging and intervention were identified.
\end{abstract}

Keyword COVID-19; Obesity; Bariatric surgery; Vaccine hesitancy

Key points

- Single-center data showed COVID-19 vaccine hesitancy in $67 \%$ of mothers post-surgery

- Most still had obesity, yet a third were unaware of the obesity risk for COVID-19

- Age, recent flu vaccine, and vaccine safety beliefs were related to hesitancy

- Expert input, understanding side effects, and health benefits may be key

Heather Strong

Heather.Strong@cchmc.org

Extended author information available on the last page of the article

\section{Introduction}

Obesity and related comorbid health conditions have played a central role in coronavirus disease 2019 (COVID-19) risk and response. Individuals with obesity are at heightened risk for experiencing more severe COVID-19 illness, hospitalizations, and progression to intensive care, as well as COVID19 related death. [1, 2] Moreover, those with obesity who recover from the primary COVID-19 infection are also more likely to experience post-acute sequelae of COVID-19 (i.e., "long COVID") compared to individuals with a healthy body mass index $\left(B M I=\mathrm{kg} / \mathrm{m}^{2}\right)$. [3] As such, in the United States (U.S.), initial vaccine distribution plans in many states prioritized individuals with obesity and/or related comorbidities for early vaccine access. [1, 4]

Encouraging evidence suggests that for those with severe obesity, undergoing bariatric surgery may reduce 
COVID-19 morbidity and mortality [5-10]. However, even with improved weight and health following bariatric surgery, many individuals may continue to present with known risk factors for severe COVID-19 (i.e., high BMI $\left[\mathrm{kg} / \mathrm{m}^{2}\right]$, unremitting Type 2 Diabetes). For example, insufficient weight loss or weight regain may result in a post-surgical BMI $\left(\mathrm{kg} / \mathrm{m}^{2}\right)$ that remains in the obese or severely obese range. [11] Understanding the interplay of surgical outcomes and COVID-19 outcomes is a critically important and emerging literature. However, even if individuals experience improved COVID-19 outcomes in connection with a history of bariatric surgery, undoubtedly surgery does not prevent risk of virus transmission. Thus, from a public health perspective, it is also imperative to understand patient COVID-19 vaccine intentions.

Widespread vaccine uptake has been essential in reducing COVID-19 related morbidity and mortality, but also in slowing transmission of the virus and the potential emergence of more life-threatening variants. [12] Yet less is known about vaccine intention in people with obesity, $[13,14]$ and specifically those who have undergone bariatric surgery. Research conducted in the general population, prior to the U.S. vaccine rollout in December 2020, [15] suggested that demographic differences, such as being female, younger, lower educational attainment, or belonging to certain ethnic minority groups were associated with greater vaccine hesitancy. [16-18] Individual attitudes and beliefs, including hesitancy about vaccines in general, uncertainty about COVID-19 vaccine safety, and apprehension due to COVID-19 vaccine novelty have also been identified as factors associated with lower vaccine intention. [18-20] Similar apprehensive attitudes were observed in the only published study to date, on COVID-19 vaccine attitudes among a sample with obesity. [14] In June to October 2020, researchers surveyed adults with obesity in Canada and found that approximately $40 \%$ were vaccine hesitant and many had low confidence in a COVID-19 vaccine.

At the time data were collected for the current study (November 2020-February 2021), national surveillance estimated that approximately half of the people in the U.S. either had (18\%) or were intending (37\%) to be vaccinated. [19] Also during this time, COVID-19 daily rates in the state where most study participants lived reached the highest peak to date, with 12,973 new cases - and 209 deaths - in a day. [21, 22] Within this dynamic context, the present study aims were to examine early COVID-19 vaccine intention among a sample of women with a history of bariatric surgery. Given larger study aims, women were also mothers with children 18 years or younger under her care. Study aims also included exploration of whether early vaccine intention varied by demographic factors, BMI $\left(\mathrm{kg} / \mathrm{m}^{2}\right)$ and knowledge of obesity as a risk for severe COVID-19, as well as general vaccine beliefs and recent flu vaccine history (used as a proxy of general vaccine acceptance). [23] Factors that would increase their confidence and motivation to obtain the COVID-19 vaccine were explored. Understanding early vaccine intention and related factors for an at-risk sample is important in order to highlight potential areas of focus for improving COVID-19 vaccine uptake. Given the unprecedented nature of this global pandemic, current findings will also help expand the field's knowledge of vaccine beliefs and intentions in the context of a pandemic, which has implications for informing approaches to future large-scale disease outbreaks.

\section{Methods}

The present study uses data obtained from the Maternal Attitudes Surrounding COVID-19 (MASC) study, the aims of which were to better understand mothers' perceptions of the impact and experiences related to COVID-19 on families with obesity. The 4-month MASC recruitment window (November 2020 to February 2021) fell during the launch of the COVID-19 vaccine distribution in the U.S. yet prior to BMI $\left(\mathrm{kg} / \mathrm{m}^{2}\right)$ or obesity being prioritized for vaccine access locally. The present study focuses on a MASC study subgroup of mothers who had undergone bariatric surgery and were recruited from a research registry of bariatric surgery candidates at a single clinical center, willing to be contacted for future research $(n=452$, registry enrollment June 2016-September 2019). MASC study eligibility required mothers to have a biological child $\leq 18$ years living in the home, access to the internet and a smart device/computer, and the ability to read and speak English fluently. All women on the registry were sent an email and/or text message introducing the study. During the MASC study window, 98 mothers from the registry consented, and 94 proceeded to participate, of whom 64 had undergone bariatric surgery since enrolling in the registry.

Following completion of the informed consent process, mothers were emailed a unique study link to complete questions online via Research Electronic Data Capture (REDCap) ${ }^{\mathrm{TM}}$. [24, 25] A subset of MASC study questions focused on vaccine intention, attitudes, and beliefs were designed by the investigators and informed by the broader literature on general vaccine acceptance, intentions, and behaviors. $[23,26]$ COVID-19 vaccine intention groupings were based on 3 response options: vaccine ready ("get it as soon as possible"), undecided ("wait and see how others respond to the vaccine first and then maybe get it"), and vaccine opposed ("not get the vaccine"). Participants also reported their history of COVID-19 symptoms or diagnosis (yes/no), knowledge of obesity as a risk factor for severe COVID-19 (yes/no), flu vaccination history in the past 12 months (yes/ no), general beliefs (i.e., not COVID-19 specific) regarding 
Table 1 Knowledge, attitudes, beliefs, and vaccine intention survey questions

\begin{tabular}{|c|c|}
\hline Questions & Response Options \\
\hline $\begin{array}{l}\text { 1. Someone in the family had symptoms or was diagnosed with } \\
\text { COVID-19 }\end{array}$ & yes, no \\
\hline $\begin{array}{l}\text { 2. Prior to today, were you aware that the CDC lists obesity as a risk } \\
\text { factor for severe illness from COVID- } 19\end{array}$ & yes I did know, no I did not know \\
\hline \multicolumn{2}{|l|}{ Attitudes and Beliefs about Vaccines in General } \\
\hline $\begin{array}{l}\text { 3. In general, I believe the use of vaccines is a safe way to protect } \\
\text { against illnesses }\end{array}$ & strongly agree, agree, disagree, strongly disagree \\
\hline $\begin{array}{l}\text { 4. In general, I believe vaccines are effective in protecting against ill- } \\
\text { nesses }\end{array}$ & strongly agree, agree, disagree, strongly disagree \\
\hline 5. Did you get a flu vaccine in the past 12 months? & yes, no \\
\hline \multicolumn{2}{|l|}{ Attitudes, Beliefs, and Intentions COVID-19 Vaccine } \\
\hline \multirow[t]{2}{*}{ 6. If a COVID-19 vaccine was made available to you, would you... } & get it as soon as possible \\
\hline & $\begin{array}{l}\text { wait to see how others respond to the vaccine first then maybe get it } \\
\text { not get the vaccine }\end{array}$ \\
\hline \multirow{7}{*}{$\begin{array}{l}\text { 7. What would make you more confident about the safety and effective- } \\
\text { ness of a COVID-19 vaccine? (check all that apply) }\end{array}$} & if my doctor recommended it for me \\
\hline & $\begin{array}{l}\text { if it is recommended by national health experts or organizations (e.g., } \\
\text { CDC, health department) }\end{array}$ \\
\hline & if national or local government officials recommend it \\
\hline & If my friends or family members get the vaccine \\
\hline & $\begin{array}{l}\text { if there are no reports of negative side effects after others begin getting } \\
\text { the vaccine }\end{array}$ \\
\hline & $\begin{array}{l}\text { nothing would make me confident about the safety and effectiveness of } \\
\text { the COVID-19 vaccine }\end{array}$ \\
\hline & other-explain \\
\hline \multirow{6}{*}{$\begin{array}{l}\text { 8. What are some things that would motivate you to get the COVID-19 } \\
\text { vaccine? (check all that apply) }\end{array}$} & if my job requires me to get the vaccine \\
\hline & I have a child or older family member living in my home \\
\hline & $\begin{array}{l}\text { I or someone in my home has a compromised immune system or } \\
\text { chronic health condition }\end{array}$ \\
\hline & if it is widely available and convenient \\
\hline & nothing would motivate me to get the COVID-19 vaccine \\
\hline & other explain \\
\hline
\end{tabular}

vaccine safety and effectiveness (strongly agree/agree/ disagree/strongly disagree), as well as factors that would increase confidence and motivation to obtain a COVID-19 vaccine. Questions from this 8-item survey on beliefs, intention, knowledge, and virus exposure are shown in Table 1. Additional self-reported information included demographics (race/ethnicity, education, partnership status, age, number of children in the home), height and weight $\left(B M I=\mathrm{kg} / \mathrm{m}^{2}\right)$, and bariatric surgery date (used to calculate time since surgery). Participants were compensated $\$ 10$. This study was approved by the Cincinnati Children's Hospital Medical Center Institutional Review Board.

Descriptive statistics were used to summarize data. Given our interest in all three vaccine intention groups (vaccine ready, undecided, vaccine opposed) and small sample sizes, univariate analyses were completed. To explore relationships between vaccine intention groups and variables of interest, one-way analyses of variance (ANOVA) and Pearson's $x^{2}$ tests were conducted for continuous and categorical variables, respectively, with post hoc analyses completed for significant ( $p<0.05$ [2-tailed]) omnibus tests. Tukey's honestly significant difference (HSD) examined all pairwise comparisons in ANOVAs. For $x^{2}$ tests, adjusted standardized residuals were used [27] with the Benjamini-Hochberg adjustment method applied to control for familywise Type 1 error. [28] There were no missing data for variables included in the analyses. All analyses were conducted using IBM SPSS Statistics software (version 26; Armonk, NY).

\section{Results}

Table 2 details sample characteristics and all outcomes of interest for the total sample, and by vaccine intention group. Consistent with national data characterizing adults undergoing bariatric surgery, [29] self-reported race and ethnicity were primarily White/European (61.9\%) and non-Hispanic/ Latinx (98.4\%). Women were approximately 39 years of 
Table 2 Sample characteristics, knowledge of obesity risk for COVID-19, history of flu vaccination, and general vaccine beliefs by COVID-19 vaccine intention group

\begin{tabular}{|c|c|c|c|c|c|c|}
\hline & All & Vaccine ready & Undecided & Vaccine opposed & $F / x^{2 \mathrm{a}}$ & $p$ \\
\hline Female, $N(\%)$ & $64(100)$ & $21(32.8)$ & $28(43.8)$ & $15(23.4)$ & N/A & \\
\hline Age (years), $M(S D)$ & $39.3(6)$ & $42.6(6.2)$ & $38.4(5)$ & $36.4(5.6)$ & 6.08 & .004 \\
\hline Race $(N=63), n(\%)^{\mathrm{b}}$ & & & & & 3.03 & .22 \\
\hline White/European & $39(60.9)$ & $15(71.4)$ & $18(64.3)$ & $6(40)$ & & \\
\hline Black/African American & $20(31.2)$ & $4(19.0)$ & $9(32.1)$ & 7 (46.7) & & \\
\hline Asian/Asian American & $1(1.6)$ & $1(4.8)$ & 0 & 0 & & \\
\hline Bi-racial/Multi-racial & $3(4.7)$ & $1(4.8)$ & $1(3.6)$ & $1(6.6)$ & & \\
\hline Unknown/Not reported & $1(1.6)$ & & & $1(6.6)$ & & \\
\hline Ethnicity $(N=61), n(\%)$ & & & & & N/A & \\
\hline Hispanic/Latinx & $1(1.6)$ & 0 & $1(3.6)$ & 0 & & \\
\hline Partnership Status, $n(\%)$ & & & & & 6.45 & .04 \\
\hline Single/Unmarried & $22(34.4)$ & $3(14.3)$ & $11(39.3)$ & $8(53.3)$ & & \\
\hline Living with partner/Married & $42(65.6)$ & $18(85.7)$ & $17(60.7)$ & $7(46.7)$ & & \\
\hline Number of children ( $\leq 18$ years) in home, $M(S D)$ & $2.3(1.3)$ & $1.9(.7)$ & $2.4(1.4)$ & $2.5(1.6)$ & 1.15 & .32 \\
\hline Highest Education, $n(\%)$ & & & & & 3.66 & .45 \\
\hline$\leq$ High School Diploma/GED & $22(34.4)$ & $7(33.3)$ & $9(32.1)$ & $6(40)$ & & \\
\hline Undergraduate degree/Vocational training & $27(42.2)$ & $7(33.3)$ & $15(53.6)$ & $5(33.3)$ & & \\
\hline Graduate-/Professional degree & $15(23.4)$ & $7(33.3)$ & $4(14.3)$ & $4(26.7)$ & & \\
\hline $\mathrm{BMI}\left(\mathrm{kg} / \mathrm{m}^{2}\right), M(S D)$ & $36.9(8.1)$ & $36(7.3)$ & $37(8.5)$ & $38.1(8.7)$ & .29 & .75 \\
\hline Healthy weight ${ }^{\mathrm{c}}, n(\%)$ & $2(3.1)$ & $2(9.5)$ & 0 & 0 & & \\
\hline Overweight $^{\mathrm{c}}, n(\%)$ & $11(17.2)$ & $2(9.5)$ & $6(21.4)$ & $3(20)$ & & \\
\hline Obesity class $\mathrm{I}^{\mathrm{c}}, n(\%)$ & $15(23.4)$ & $6(28.6)$ & $7(25)$ & $2(13.3)$ & & \\
\hline Obesity class $\geq \mathrm{II}^{\mathrm{c}}, n(\%)$ & $36(56.3)$ & $11(52.4)$ & $15(53.6)$ & $10(66.7)$ & & \\
\hline History of COVID-19 diagnosis or symptoms ${ }^{\mathrm{d}}, n(\%)$ & $12(18.8)$ & $7(33.3)$ & $2(7.1)$ & $3(20)$ & N/A & \\
\hline Had knowledge of obesity as a risk for COVID- $19^{\mathrm{d}}, n(\%)$ & $41(64.1)$ & $14(66.7)$ & $17(60.7)$ & $10(66.7)$ & .24 & .89 \\
\hline Received flu vaccine (past 12 months) ${ }^{\mathrm{e}}, n(\%)$ & $39(60.9)$ & $17(81)$ & $17(60.7)$ & $5(33.3)$ & 8.34 & .02 \\
\hline \multicolumn{7}{|l|}{ General belief vaccines are safe ${ }^{e}$, } \\
\hline$n(\%)$ & $51(79.7)$ & $20(95.2)$ & $23(82.1)$ & $8(53.3)$ & 9.68 & .01 \\
\hline General belief vaccines are effective ${ }^{\mathrm{e}}, n(\%)$ & $56(87.5)$ & $20(95.2)$ & $25(89.3)$ & $11(73.3)$ & 3.98 & .14 \\
\hline
\end{tabular}

${ }^{\mathrm{a}}$ Chi-square tests $\left(x^{2}\right)$ were completed for percentages and ANOVAs $(F)$ were completed for mean values to test differences among vaccine intention groups $(d f=2)$

${ }^{b}$ Dichotomized as White/European vs Non-White/European for chi-square analysis

${ }^{\mathrm{c}}$ BMI categorized as healthy weight at $18.5-<25 \mathrm{~kg} / \mathrm{m}^{2}$, overweight at $25-<30 \mathrm{~kg} / \mathrm{m}^{2}$, obesity class I at $30-<35 \mathrm{~kg} / \mathrm{m}^{2}$, obesity class II at $\geq 35 \mathrm{~kg} / \mathrm{m}^{2}$

${ }^{\mathrm{d}}$ Number of participants who responded "yes"; variable represented in analysis as "yes" and "no"

"Number of participants who responded, "strongly agree" or "agree"; variable dichotomized in analysis as "strongly agree"/ "agree" and "strongly disagree"/ "disagree"

age (range 25.9-54.7 years), with most residing with a partner $(65.6 \%)$ and 2 biological children $\leq 18$ years (range 1-6 children) and having completed post-secondary education/training (65.6\%). Mean time since bariatric surgery was 19.6 months ( $S D=10.9$; range $<1$ month -47 months) with the majority of mothers meeting criteria for obesity $\left(79.7 \% \geq\right.$ Class I $\left.\left[\mathrm{BMI}=\geq 30 \mathrm{~kg} / \mathrm{m}^{2}\right]\right)$ if not severe obesity $\left(56.3 \% \geq\right.$ Class II $\left.\left[\mathrm{BMI}=\geq 35 \mathrm{~kg} / \mathrm{m}^{2}\right]\right)$. Nearly 1 in 5 reported a previous diagnosis or symptoms of COVID-19.

Approximately 1 in 3 women were in the vaccine-ready group, $(n=21 ; 32.8 \%)$, with the majority either in the undecided $(n=28 ; 43.8 \%)$ or vaccine-opposed $(n=15 ; 23.4 \%)$ group (Fig. 1). Vaccine intention significantly varied between groups based on age $(p=0.004)$, with the undecided and vaccineopposed groups being significantly younger (undecided $p=0.03$; vaccine opposed $p=0.01$ ) relative to the vaccine-ready group. Vaccine intention was significantly associated with partnership status $(p=0.04)$, with higher than expected rates of married/residing partner participants in the vaccine-ready group $(p=0.02)$. Vaccine intention was unrelated to race (defined as White/European vs. Other racial/ethnic groups), education, number of children in the home, or current BMI $\left(\mathrm{kg} / \mathrm{m}^{2}\right)$. 


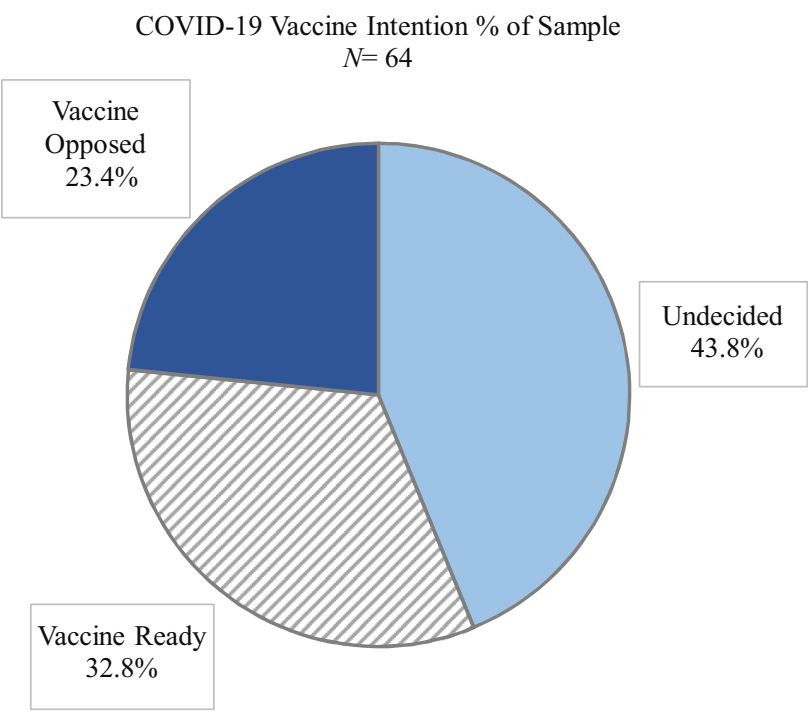

Fig. 1 Vaccine intention group based on response to item: "If a COVID-19 vaccine was made available to you would you... get it as soon as possible (vaccine ready), wait and see how others respond to vaccine and then maybe get it (undecided), or not get the vaccine (not vaccine opposed)."

COVID-19 vaccine intention was associated with a general belief that vaccines are safe $(p=0.008)$ and obtaining a flu vaccine in the past 12 months $(p=0.02)$, but unrelated to a general belief that vaccines are effective or to knowledge of obesity increasing risk for severe COVID-19. Post hoc analyses indicated that belief that vaccines are safe was higher than expected in the vaccine-ready group $(p=0.004)$ and lower in the vaccine-opposed group $(p=0.03)$. Similarly, flu vaccination rates were higher than expected in the vaccine-ready group $(p=0.02)$ and lower in the vaccineopposed group $(p=0.01)$.

The vaccine-ready and undecided groups endorsed multiple factors that would increase their confidence in COVID19 vaccine safety and effectiveness, including clinician and other health expert recommendations, and for the undecided group, reports of no side effects were also important (Fig. 2). For the vaccine-ready and undecided groups, motivating factors to get a COVID-19 vaccine included an employer mandate or a compromised immune system for self or others in the home (Fig. 3). In stark contrast, the majority of the vaccine-opposed group reported "nothing" would increase their confidence or motivate them to obtain a COVID-19 vaccine.

\section{Discussion}

While preliminary, these data provide an early snapshot of prominent vaccine hesitancy in a clinical sample of mothers post-bariatric surgery during the peak of new COVID19 cases and deaths both locally and nationally, as well as during the initial COVID-19 vaccine distribution phase in the U.S. $[15,21,22]$ Two in 3 were unsure $(43.8 \%)$ or not planning to get the vaccine (23.4\%), numbers fairly consistent with national views during the same time period. [19]
Fig. 2 Factors contributing to increased confidence summarized as \% endorsed by each vaccine intention group
What would make you more confident about the safety and effectiveness of a COVID-19 vaccine? (check all that apply)

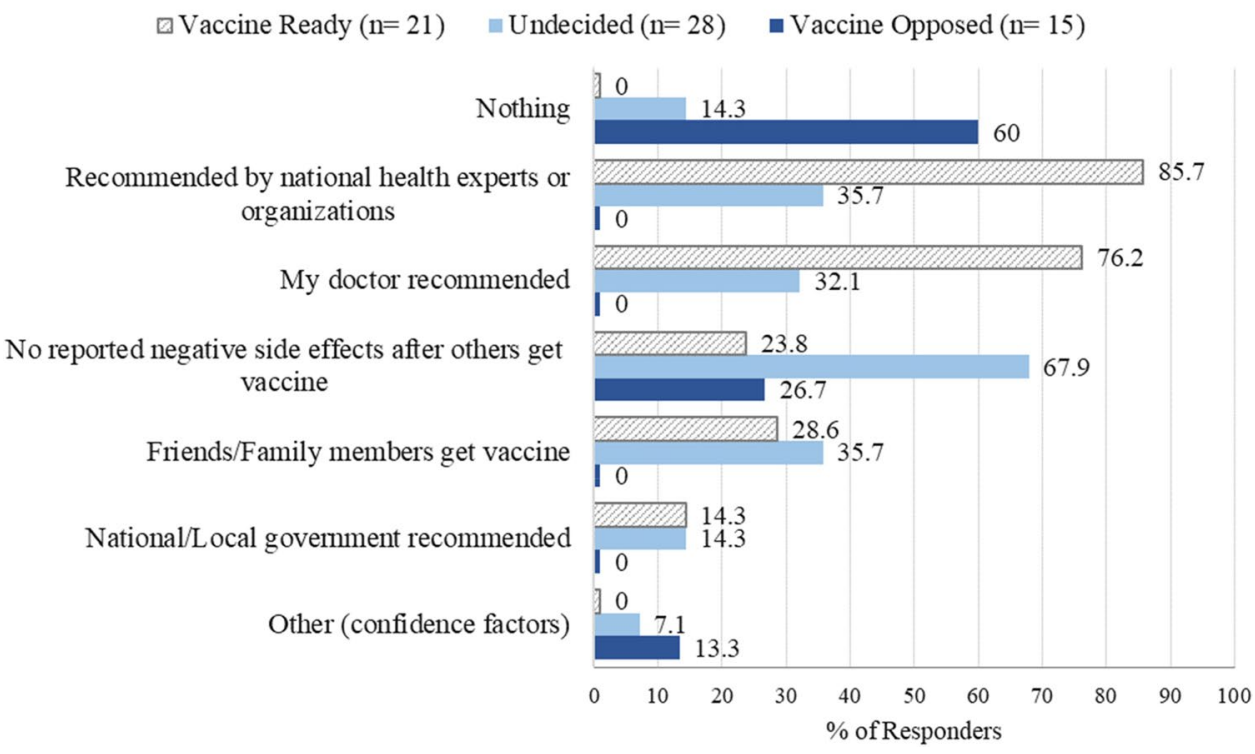

Other (confidence factors) responses included follow-up qualitative responses [ $n=4]$, the theme across groups was a desire to see "long term" data demonstrating no side effects. 
Fig. 3 Factors contributing to motivation to get the COVID19 vaccine summarized as $\%$ endorsed by each vaccine intention group
What are some things that would motivate you to get a COVID-19 vaccine? (check all that apply)

๒Vaccine Ready $(\mathrm{n}=21) \quad \square$ Undecided $(\mathrm{n}=28) \quad$ Vaccine Opposed $(\mathrm{n}=15)$

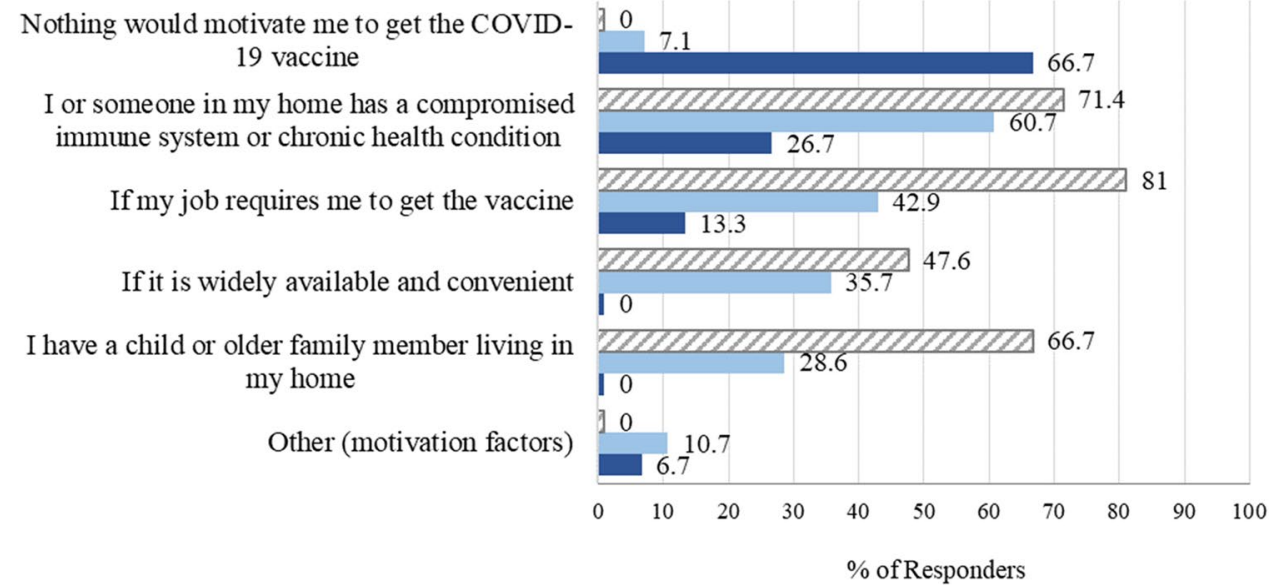

Other (motivation factors) responses included follow-up qualitative responses $[n=4]$, the theme across groups was "no negative side effects" reported.
However, unique to the present sample is not only a postbariatric surgery context, but a high prevalence of persisting obesity, if not severe obesity. Consistent with national data, [30] the majority had knowledge that their obesity placed them at risk for severe COVID-19 morbidity and mortality. Yet that 1 in $3(35.9 \%)$ still lacked that knowledge during the "third wave" in the United States when the daily number of new cases reached their peak is concerning. Although there is some evidence to suggest bariatric surgery may contribute to reduced morbidity and mortality, the potential protective mechanism of bariatric surgery is not fully understood in individuals who persist with obesity. [5-7, 9, 10] Also of note, despite the positive role that bariatric surgery may have in reducing severe COVID-19 outcomes, it does not protect against virus transmission, which remains a critical public health concern.

At the time of this writing, in the U.S. and locally, rates of COVID-19 vaccinations were $<50 \%$. [22, 31] With the growing spread of the COVID-19 Delta variant, largely among those who are unvaccinated, it has been recommended that individuals return to wearing masks in public, with a concern for a return to increased public restrictions. $[21,32]$ The present findings illustrate the potential for a clinical subpopulation at continued high risk, as well as highlight factors to tailor COVID-19 vaccine acceptance messaging in bariatric surgery care. Given many acknowledged their trust in recommendations of their medical providers and other health experts, bariatric surgery clinicians may be positioned to have a positive impact on vaccine uptake for some hesitant patients, including younger, female patients who may be more vaccine hesitant. Knowledge of patients' recent flu vaccination history may also suggest when targeted discussion about vaccine beliefs would prove timely.

To increase confidence and motivation, clinicians might be primed to explain clinical trial processes and outcomes, including anecdotal experiences of short-term and tolerable side effects. Providing education on how and where to access the vaccine and that it is provided at no cost will also reduce misinformation. Counseling hesitant patients in ways that resonate with their values may prove helpful, noting protective benefits of vaccination for their health and the health of loved ones. Fortunately, bariatric surgery team members have multiple opportunities to engage patients during followup across the first post-bariatric surgery year, but clinicians should consider additional means of patient outreach (i.e., mailed newsletters and MyChart messaging) given the likelihood of decreased clinic attendance over time. [33] Moreover, considering these data characterize mothers, there are broader intergenerational implications, as parents typically are vaccine decision-makers for their children. [23] Finally, it is important to acknowledge that some individuals (i.e., vaccine-opposed group) will be particularly difficult to persuade to get a COVID-19 vaccination. As such, continued research and public engagement are important in order to understand the perspective of hard-to-reach populations.

To our knowledge, these are the first data characterizing early COVID-19 vaccine hesitancy within a sample of 
patients who underwent bariatric surgery, while also adding to emerging research on vaccine intention among individuals with obesity who are an identified high-risk population. This is particularly timely given the resurgence of COVID-19 hospitalizations due to new disease variants and as indications and parameters for a booster shot are debated. [31, 34] While novel, this study is not without limitations. Due to study design, current data are limited by sample size and in that they are from a single medical center in the Midwestern U.S. and represent only mothers who were reachable during a brief study window (November 2020 to February 2021) during the initial U.S. distribution of a COVID-19 vaccine. Further, the sample only included mothers with residing biological children ( $\leq 18$ years), which may have contributed to a sample younger than the average age of bariatric surgery patients in the U.S. (i.e., 44 years old). $[29,35]$ Also, because we recruited from an existing patient registry limited to biological mothers, data do not include men, or women without biological children. All these factors may impact the generalizability of the current findings to the broader bariatric surgery population. Though this sample was similar to the larger U.S. bariatric surgery population in that it was predominantly Non-Hispanic/Latinx, White, indeed the sample reflects a systemic disparity in the larger patient population. [29] As such, given the sample's limited racial and ethnic diversity, data may not fully capture the experience of all mothers who have undergone bariatric surgery. In addition, while the majority of study participants (63\%) completed surveys during the initial vaccine rollout in the U.S., what has unfolded since has unexpectedly become an ever-changing landscape of COVID-19. As a result, intentions and beliefs may have changed for mothers after study completion. Research is critically needed with larger and more diverse samples (e.g., non-White, lower socioeconomic status, non-English speaking) with obesity to fully capture this evolving crisis and dynamic vaccine hesitancy and uptake trends over time. As recently noted by others, additional work should also consider other potential obesity-specific contributors to COVID-19 vaccine hesitancy, including uncertainty about vaccine effectiveness [13] and the effects of obesity-specific clinical- and public health messaging on experiences of weight bias and stigma and vaccine decision-making. [36]

\section{Conclusions}

The current pandemic presents an intersection of two public health crises, COVID-19 and obesity. As the COVID-19 vaccine campaign continues and public health initiatives work to encourage COVID-19 vaccination among those who are hesitant, targeted and specific efforts may be needed to ensure COVID-19 vaccine uptake in groups with obesity, including those who have undergone bariatric surgery.

Acknowledgements The authors would like to thank the women who participated in this study. We would also like to acknowledge the clinical staff at UC Health Weight Loss Center (Cincinnati, OH): Jon Thompson, MD, and Diana Harris; and additional members of the research team at Cincinnati Children's Hospital Medical Center: Faye Doland, BS, and Kristina Decker, PhD. We would also like to acknowledge the Center for Clinical and Translational Science and Training which provided institutional grant support (2UL1TR001425-05A1) for the REDCap data collection system.

Funding This project and Dr. Strong's effort were supported by a postdoctoral training grant from the National Institutes of Health (NIH), National Institutes of Diabetes and Digestive and Kidney Diseases (T32DK063929-18S1).

\section{Declarations}

Ethics Approval All procedures performed in studies involving human participants were in accordance with the ethical standards of the institutional and/or national research committee and with the 1964 Helsinki Declaration and its later amendments or comparable ethical standards.

Informed Consent Informed consent was obtained from all individual participants included in the study.

Conflict of Interest The authors declare no competing interests.

\section{References}

1. Popkin BM, Du S, Green WD, Beck MA, Algaith T, Herbst CH, et al. Individuals with obesity and COVID-19: a global perspective on the epidemiology and biological relationships. Obes Rev. 2020;21(11):e13128. https://doi.org/10.1111/obr.13128.

2. Yang J, Hu J, Zhu C. Obesity aggravates COVID-19: a systematic review and meta-analysis. J Med Virol. 2021;93(1):257-61. https://doi.org/10.1002/jmv.26237.

3. Aminian A, Bena J, Pantalone KM, Burguera B. Association of obesity with post-acute sequelae of COVID-19 (PASC). Diabetes Obes Metab. 2021;23:2183-8. https://doi.org/10.1111/dom. 14454.

4. Landsverk G. Check to see if your BMI is in the 'obese' range, because you may be able to get an early COVID-19 vaccine. Updated March 4, 2021. Accessed March 11, 2021. https://www. businessinsider.com/how-to-check-if-obesity-qualifies-for-covid19-vaccine-2021-2? $\mathrm{r}=\mathrm{US} \& \mathrm{IR}=\mathrm{T}$.

5. Aminian A, Fathalizadeh A, Tu C, Butsch WS, Pantalone KM, Griebeler ML, et al. Association of prior metabolic and bariatric surgery with severity of coronavirus disease 2019 (COVID-19) in patients with obesity. Surg Obes Relat Dis. 2021;17(1):208-14. https://doi.org/10.1016/j.soard.2020.10.026.

6. Bel Lassen P, Poitou C, Genser L, Marchelli F, Aron-Wisnewsky J, Ciangura C, et al. COVID-19 and its severity in bariatric surgery-operated patients. Obesity (Silver Spring). 2021;29(1):24-8. https://doi.org/10.1002/oby.23026.

7. Marchesi F, Valente M, Riccò M, Rottoli M, Baldini E, Mecheri F, et al. Effects of bariatric surgery on COVID-19: a multicentric study from a high incidence area. Obes Surg. 2021;31(6):247788. https://doi.org/10.1007/s11695-020-05193-w. 
8. Conceição E, de Lourdes M, Ramalho S, Félix S, Pinto-Bastos A, Vaz AR. Eating behaviors and weight outcomes in bariatric surgery patients amidst COVID-19. Surg Obes Relat Dis. 2021;17(6):1165-74. https://doi.org/10.1016/j.soard.2021.02.025.

9. Uccelli M, Cesana GC, De Carli SM, Ciccarese F, Oldani A, Zanoni AAG, et al. COVID-19 and obesity: is bariatric surgery protective? Retrospective analysis on 2145 patients undergone bariatric-metabolic surgery from high volume center in Italy (Lombardy). Obes Surg. 2021;31(3):942-8. https://doi.org/10.1007/s11695-020-05085-z.

10 Iannelli A, Bouam S, Schneck AS, Frey S, Zarca K, Gugenheim $\mathrm{J}$, et al. The impact of previous history of bariatric surgery on outcome of COVID-19. A nationwide medico-administrative french study. Obes Surg. 2021;31(4):1455-63. https://doi.org/10.1007/ s11695-020-05120-z.

11. El Ansari W, Elhag W. Weight regain and insufficient weight loss after bariatric surgery: definitions, prevalence, mechanisms, predictors, prevention and management strategies, and knowledge gaps-a scoping review. Obes Surg. 2021;31:1755-66. https:// doi.org/10.1007/s11695-020-05160-5.

12. Centers for Disease Control and Prevention. Vaccine safety \& monitoring: Safety of COVID-19 vaccines. Updated March 30, 2021. Accessed April 1 2021. https://www.cdc.gov/coronavirus/ 2019-ncov/vaccines/safety/safety-of-vaccines.html.

13. Townsend MJ, Kyle TK, Stanford FC. COVID-19 vaccination and obesity: optimism and challenges. Obesity (Silver Spring). 2021;29(4):634-5. https://doi.org/10.1002/oby.23131.

14. Vallis M, Glazer S. Protecting individuals living with overweight and obesity: attitudes and concerns toward COVID-19 vaccination in Canada. Obesity (Silver Spring). 2021;29(7):1128-37. https:// doi.org/10.1002/oby.23182.

15. U.S. Food and Drug Administration. Emergency use authorization. Updated July 30, 2021. Accessed September 11, 2021. https://www. fda.gov/emergency-preparedness-and-response/mcm-legal-regul atory-and-policy-framework/emergency-use-authorization.

16. Malik AA, McFadden SM, Elharake J, Omer SB. Determinants of COVID-19 vaccine acceptance in the US. EClinicalMedicine. 2020;26:100495. https://doi.org/10.1016/j.eclinm.2020.100495.

17. Robinson E, Jones A, Lesser I, Daly M. International estimates of intended uptake and refusal of COVID-19 vaccines: a rapid systematic review and meta-analysis of large nationally representative samples. Vaccine. 2021;39(15):2024-34. https://doi.org/10. 1016/j.vaccine.2021.02.005.

18. Al-Amer R, Maneze D, Everett B, Montayre J, Villarosa AR, Dwekat E, et al. COVID-19 vaccination intention in the first year of the pandemic: a systematic review. J Clin Nurs. 2021. https:// doi.org/10.1111/jocn.15951s

19. Hamel L, Sparks G, Broadie M. KFF COVID-19 vaccine monitor: February 2021. Updated February 26, 2021. Accessed March 3, 2021. https://www.kff.org/coronavirus-covid-19/poll-finding/kffcovid-19-vaccine-monitor-february-2021/

20. Thunstrom L, Ashworth M, Finnoff D, Newbold S. Hesitancy towards a COVID-19 vaccine and prospects for herd immunity. SSRN., 2021:1-50. Updated June 30, 2020. Accessed March 3. https://doi.org/10.2139/ssrn.3593098

21. Centers for Disease Control and Prevention. COVID data tracker: compare trends in COVID-19 cases and deaths in the US. Accessed July 28, 2021. https://covid.cdc.gov/covid-data-track er/\#compare-trends_newdeaths.

22. State of Ohio Department of Health. State of Ohio COVID-19 dashboard. Updated August 3, 2021. Accessed August 32021. https://coronavirus.ohio.gov/wps/portal/gov/covid-19/dashboards/ covid-19-vaccine/covid-19-vaccination-dashboard.

23. Brewer NT, Chapman GB, Rothman AJ, Leask J, Kempe A. Increasing vaccination: putting psychological science into action. Psychol Sci Public Interest. 2017;18(3):149-207. https://doi.org/ 10.1177/1529100618760521.

24. Harris PA, Taylor R, Thielke R, Payne J, Gonzalez N, Conde JG. Research electronic data capture (REDCap)-a metadata-driven methodology and workflow process for providing translational research informatics support. J Biomed Inform. 2009;42(2):37781. https://doi.org/10.1016/j.jbi.2008.08.010.

25. Harris PA, Taylor R, Minor BL, Elliott V, Fernandez M, O’Neal $\mathrm{L}$, et al. The REDCap consortium: building an international community of software platform partners. J Biomed Inform. 2019;95:103208. https://doi.org/10.1016/j.jbi.2019.103208.

26. Sarathchandra D, Navin MC, Largent MA, McCright AM. A survey instrument for measuring vaccine acceptance. Prev Med. 2018;109:1-7. https://doi.org/10.1016/j.ypmed.2018.01.006.

27. Sharpe D. Chi-square test is statistically significant: now what? Practical Assessment, Research, and Evaluation. 2015; 20(8). https://doi.org/10.7275/tbfa-x148

28. Benjamini Y. Discovering the false discovery rate. J R Stat Soc Series B Stat Methodol. 2010;72(4):405-16. https://doi.org/10. 1111/j.1467-9868.2010.00746.x.

29. Campos GM, Khoraki J, Browning MG, Pessoa BM, Mazzini GS, Wolfe L. Changes in utilization of bariatric surgery in the United States from 1993 to 2016. Ann Surg. 2020;271(2):201-9. https:// doi.org/10.1097/sla.0000000000003554.

30. Schoeni RF, Wiemers EE, Seltzer JA, Langa KM. Association between risk factors for complications from COVID-19, perceived chances of infection and complications, and protective behavior in the US. JAMA Netw Open. 2021;4(3):e213984. https://doi.org/ 10.1001/jamanetworkopen.2021.3984.

31. Centers for Disease Control and Prevention. Covid-19 data tracker: Covid-19 vaccinations in the United States. Updated July 28, 2021. Accessed August 3, 2021. https://covid.cdc.gov/coviddata-tracker/\#vaccinations.

32. Centers for Disease Control and Prevention. When you've been fully vaccinated. Updated July 27, 2021. Accessed August 25 2021. https://www.cdc.gov/coronavirus/2019-ncov/vaccines/ fully-vaccinated.html

33. Goldenshluger A, Elazary R, Cohen MJ, Goldenshluger M, Ben-Porat T, Nowotni J, et al. Predictors for adherence to multidisciplinary follow-up care after sleeve gastrectomy. Obes Surg. 2018;28(10):3054-61. https://doi.org/10.1007/ s11695-018-3296-7.

34. Krause PR, Fleming TR, Peto R, Longini IM, Figueroa JP, Sterne JA, et al. Considerations in boosting COVID-19 vaccine immune responses. The Lancet. 2021:02046-8. https://doi.org/10.1016/ S0140-6736(21)02046-8

35. Alalwan AA, Friedman J, Park H, Segal R, Brumback BA, Hartzema AG. US national trends in bariatric surgery: a decade of study. Surgery. 2021;170(1):13-7. https://doi.org/10.1016/j. surg.2021.02.002.

36. Pearl RL, Schulte EM. Weight bias during the COVID-19 pandemic. Curr Obes Rep. 2021;10(2):181-90. https://doi.org/10. 1007/s13679-021-00432-2.

Publisher's Note Springer Nature remains neutral with regard to jurisdictional claims in published maps and institutional affiliations. 


\section{Authors and Affiliations}

\section{Heather Strong ${ }^{1}\left[\right.$. Jennifer Reiter-Purtill ${ }^{1}$. Taylor Howarth ${ }^{1} \cdot$ Lisa West-Smith ${ }^{2} \cdot$ Meg H. Zeller $^{1,3}$}

1 Division of Behavioral Medicine and Clinical Psychology, Cincinnati Children's Hospital Medical Center, 3333 Burnet Avenue, Cincinnati, OH 45229, USA

2 Department of Psychiatry and Behavioral Neuroscience \& Department of Surgery, University of Cincinnati College of Medicine, 3230 Eden Avenue, Cincinnati, OH 45267, USA
3 Department of Pediatrics, University of Cincinnati College of Medicine, 3230 Eden Avenue, Cincinnati, OH 45267, USA 